\title{
Pharmacokinetic/Pharmacodynamic Profiles of Tiamulin in an Experimental Intratracheal Infection Model of Mycoplasma gallisepticum
}

\author{
Xia Xiao ${ }^{1,2 \dagger}$, Jian Sun ${ }^{1 \dagger}$, Tao Yang ${ }^{1}$, Xi Fang ${ }^{1}$, Jie Cheng ${ }^{1}$, Yan Q. Xiong ${ }^{3,4}$ and Ya-Hong Liu ${ }^{1,2 *}$ \\ ${ }^{1}$ National Reference Laboratory of Veterinary Drug Residues (SCAU), College of Veterinary Medicine, South China \\ Agricultural University, Guangzhou, China, ${ }^{2}$ Jiangsu Co-Innovation Centre for Prevention and Control of Important Animal \\ Infectious Diseases and Zoonoses, Yangzhou, Jiangsu, China, ${ }^{3}$ Los Angeles Biomedical Research Institute, Harbor-UCLA \\ Medical Center, Torrance, CA, USA, ${ }^{4}$ David Geffen School of Medicine, UCLA, Los Angeles, CA, USA
}

\section{OPEN ACCESS}

Edited by:

Zong-Hui Yuan,

Huazhong Agricultural University,

China

Reviewed by:

Ayhan Filazi,

Ankara University, Turkey Begum Yurdakok Dikmen,

Ankara University, Turkey

${ }^{*}$ Correspondence:

Ya-Hong Liu

gale@scau.edu.cn

tXia Xiao and Jian Sun contributed equally to this work.

Specialty section: This article was submitted to Veterinary Pharmacology and Toxicology,

a section of the journal

Frontiers in Veterinary Science

Received: 20 July 2016 Accepted: 23 August 2016 Published: 06 September 2016

Citation:

Xiao $X$, Sun J, Yang T, Fang $X$, Cheng J, Xiong YQ and Liu Y-H (2016) Pharmacokinetic/ Pharmacodynamic Profiles of

Tiamulin in an Experimental Intratracheal Infection Model of Mycoplasma gallisepticum. Front. Vet. Sci. 3:75. doi: 10.3389/fvets.2016.00075
Mycoplasma gallisepticum is the most important pathogen in poultry among four pathogenic Mycoplasma species. Tiamulin is a pleuromutilin antibiotic that shows a great activity against $M$. gallisepticum and has been approved for use in veterinary medicine particularly for poultry. However, the pharmacokinetic/pharmacodynamics (PK/PD) profiles of tiamulin against $M$. gallisepticum are not well understood. Therefore, in the current studies, we investigated the in vivo PK/PD profiles of tiamulin using a well-established experimental intratracheal infection model of $M$. gallisepticum. The efficacy of tiamulin against $M$. gallisepticum was studied in 8-day-old chickens after intramuscular (i.m.) administration at 10 doses between 0-80 mg/kg. Liquid chromatography-tandem mass spectrometry (LC-MS/MS) was used to evaluate the PK parameters of tiamulin following i.m. administration at doses of 5, 40, and $80 \mathrm{mg} / \mathrm{kg}$ in Mycoplasma gallisepticum-infected neutropenic chickens. Real-time PCR (RT-PCR) was used for quantitative detection of M. gallisepticum. The MIC of tiamulin against M. gallisepticum strain S6 was $0.03 \mu \mathrm{g} / \mathrm{mL}$. The PK/PD index, $\mathrm{AUC}_{24 \mathrm{~h}} / \mathrm{MIC}$, correlated well with the in vivo antibacterial efficacy. The in vivo data suggest that animal dosage regimens should supply $\mathrm{AUC}_{24 h} / \mathrm{MIC}$ of tiamulin of $382.68 \mathrm{~h}$ for $2 \log _{10}$ ccu equivalents $M$. gallisepticum reduction. To attain that goal, the administered dose is expected to be $45 \mathrm{mg} / \mathrm{kg}$ b.w. for treatment of $M$. gallisepticum infection with an $\mathrm{MIC}_{90}$ of $0.03 \mu \mathrm{g} / \mathrm{mL}$.

Keywords: tiamulin, M. gallisepticum, in vivo PK/PD, chicken

\section{INTRODUCTION}

Mycoplasma gallisepticum (M. gallisepticum), a multi-host pathogen, is the most pathogenic agent of chronic respiratory disease (CRD) in poultry and turkey $(1,2)$. It can also be transmitted from poultry to house finches as well as other similar species and cause outbreaks of upper respiratory disease (3). Because it produces vast losses in the poultry industry, M. gallisepticum is considered as the most economically important pathogen of the four pathogenic Mycoplasma species (4).

Current means of controlling M. gallisepticum infections among avian species include biosurveillance practices, vaccines, and medication (5). Despite control measures, M. gallisepticum may be present in chick flocks that are maintained for long growing periods with minimal biosecurity. Some live attenuated M. gallisepticum vaccines are approved for use only within the commercial egg layer 
industry (6). The effectiveness of other subunit vaccines may be limited due to efficacy and associated costs (7). Although some vaccines are effective, $M$. gallisepticum outbreaks within vaccinated flocks has been observed (7), suggesting better medication control are required.

Antibiotics have been extensively used in the areas with intensive and varied population of poultry flocks for controlling M. gallisepticum or other pathogen infections (8). The use of antimicrobial agents has been considered as an economic method for controlling M. gallisepticum infections (8). Several kinds of antibiotics (macrolides, tetracyclines, fluoroquinolones, and pleuromutilin) have displayed good activity against $M$. gallisepti$\operatorname{cum}(9,10)$. However, it has also been noticed that antibacterial usage over time can induce resistance in this organism $(11,12)$.

Tiamulin is a semisynthetic derivative of the diterpene antibiotic pleuromutilin used in swine and poultry for treatment and prophylaxis of dysentery, pneumonia, and mycoplasmal infections (13). Although it has been used over 30 years, no significant changes of susceptibility on this pathogen were observed previously $(11,14)$. However, one recent investigation indicated that tiamulin resistant isolates were seen after tiamulin treatment (15). Therefore, it is important to study the optimal tiamulin regimen in order to maximize antibacterial activity and to prevent emergence of resistance. It is well-known that pharmacokinetic (PK) and pharmacodynamics (PDs) profiles of antibiotics provide useful information in the establishment of optimal dose regimens for better clinical setting management and prohibit resistance emergence $(16,17)$. To our best knowledge, PK/PD profiles of tiamulin against $M$. gallisepticum are very limited. Therefore, in the current experiment, we performed in vivo PK/PD studies of tiamulin against M. gallisepticum after intramuscular (i.m.) administration. Though the i.m. administration has not been approved for tiamulin in chicks, this study would provide foundation for tiamulin injectable formulation for chicks in future. The goals of the present investigations were to: (1) evaluate the PK profiles and dose proportionality of tiamulin in an intratracheal infection animal model; (2) provide the magnitude of the PK/PD index $\mathrm{AUC}_{24 \mathrm{~h}} / \mathrm{MIC}$ for different extent of efficacies; and (3) establish a rational dosage regimen that optimizes tiamulin efficacy with respect to bacteriological and clinical outcomes.

\section{MATERIALS AND METHODS}

\section{Bacteria, Chemicals, Susceptibility Assay, and Animals}

A well-characterized M. gallisepticum standard virulent strain S6 was purchased from the Chinese Veterinary Microorganism Culture Collection Center (Beijing, China). Tiamulin Fumarate (>99\%) was kindly supplied by the Hebei Yuanzheng Pharmaceutical Company (Hebei, China). The MIC of tiamulin on the strain S6 was determined by a standard micro-dilution method according to recommended protocols $(18,19)$. Three hundred fifty Sanhuang chickens of 1-day-old weighting 35 45 g supplied by Guangdong Academy of Agricultural Sciences (Guangzhou, Guangdong, China) were used in this experiment. Birds were free of M. gallisepticum and fed with clean water and antibacterial-free fodder.

\section{In Vitro Standard DNA Preparing}

An in vitro DNA standard curve was established according to our previous report (19). Briefly, $36 \mathrm{~h}$ incubated M. gallisepticum medium was centrifuged for $10 \mathrm{~min}$ at $1,500 \mathrm{rpm}$ and then resuspending the pellet in $0.6 \mathrm{~mL}$ fresh $M$. gallisepticum medium. $0.1 \mathrm{~mL}$ of the sample was serial diluted for bacteria counting by culture method [color change unit per millimeter $(\mathrm{ccu} / \mathrm{mL})]$. Meanwhile, DNA was isolated from the sample and serial 10 -fold dilutions $\left(10^{0}-10^{-6}\right)$ prepared from the $0.6 \mathrm{~mL}$ sample with a bacteria DNA kit (Omega Bio-tek, Inc., Norcross, GA, USA). The DNA copies of M. gallisepticum were determined by real-time PCR (RT-PCR) (20). The DNA standard curve was plotted by the number of $M$. gallisepticum calculated from the culture method and cycle threshold $\left(C_{\mathrm{t}}\right)$ values obtained using RT-PCR results.

\section{Neutropenia Model}

Two days post arrival, the chicken neutropenia model published by our laboratory (19) was established in this study in order to eliminate the immunity variance of different chickens and study the efficacy of tiamulin solely, via intramuscular administration of cyclophosphamide at $60 \mathrm{mg} / \mathrm{kg}$ for 3 days (21). Birds were severely granulocytopenic (absolute leukocyte count $<1,000 / \mathrm{mm}^{3}$ ) and remained so for 8 days after the third injection of cyclophosphamide. The South China Agriculture University Animal ethics committee approved all in vivo experiments with an approved number of 2014-08. In addition, all husbandry practices and experimental operations were performed with full consideration of animal welfare.

\section{M. gallisepticum Intratracheal Infection Model}

M. gallisepticum mainly invades the respiratory system in chickens. Thus, an $M$. gallisepticum intratracheal infection model was utilized in this study according to Xia's report (19). Briefly, $24 \mathrm{~h}$ post the last dose of cyclophosphamide, $0.2 \mathrm{~mL}$ of solution containing approximately $10^{8}$ color change unit (ccu) of the M. gallisepticum strain was inoculated intratracheally to neutropenic chickens for 3 days [95\% infective dose $\left(\mathrm{ID}_{95}\right)$ for the studied strain]. Initial pathogen loading was quantified according to our established method described in our previous report (19). Briefly, at $24 \mathrm{~h}$ after the last infection dose, chickens were euthanized and trachea, air sac, and lungs were collected, homogenized in $2 \mathrm{~mL}$ PBS and centrifuged at $500 \mathrm{rpm}$ for $5 \mathrm{~min}$. An aliquot of $0.5 \mathrm{~mL}$ supernatant was used for DNA extraction with a bacterial DNA kit (Omega Bio-tek, Inc., Norcross, GA, USA) as described above. DNA copies of M. gallisepticum in these samples were measured by RT-PCR as described above. The amount of $M$. gallisepticum was calculated using the DNA copies via the in vitro standard curve.

\section{Determination of DNA Copies of M. gallisepticum Using RT-PCR}

The method determinating DNA copies of M. gallisepticum in different samples by RT-PCR were identical with our previous report (19). All RT-PCR reactions were performed on a 
BIO-RAD iQ 5 (Bio-Rad Laboratories, Inc., USA) using the SYBR premix Ex Taq ${ }^{\mathrm{TM}}$ (TaKaRa, Shiga, Japan). $C_{t}$ values were defined as the cycle number yielding a maximum value of the second derivative of the amplification curve of the sample. Samples were defined as positive when both a measurable $C_{t}$ and the expected $T_{m}\left( \pm 0.5^{\circ} \mathrm{C}\right)$ were seen. The standard samples and a negative control (elution buffer) were included in each run.

\section{Tiamulin Pharmacokinetics in Neutropenic Intratracheal Infection Model}

The infected neutropenic chickens were administrated with tiamulin intramuscularly at single doses of 5,40 , or $80 \mathrm{mg} / \mathrm{kg}$ b.w. $1.5 \mathrm{~mL}$ blood was sampled from the neck vein at 5, 10, 30 min, 1, 2, 4, 6, 8, 12, and $24 \mathrm{~h}$ after drug administration (10 chickens/time point). Blood samples were incubated immediately at room temperature for $1 \mathrm{~h}$ and then placed in $4^{\circ} \mathrm{C}$ for $2 \mathrm{~h}$ to enable clot retraction. Serum was obtained by centrifuging at $3,000 \mathrm{rpm}$ for $10 \mathrm{~min}$ and frozen at $-20^{\circ} \mathrm{C}$ immediately until analysis within 2 weeks. Tiamulin was extracted from serum by acetonitrile with a proportion of 2:1. Concentrations of tiamulin in serum were determined via a high-performance liquid chromatography-tandem mass spectrometry (LC-MS/ MS) method, which was developed by our group and reported previously $(22,23)$. The recovery and precision were calculated by analysis of spiked samples at three concentration levels (5 replicates of each concentration). Mean recoveries of tiamulin that spiked at three concentration levels were in the range of $86.0-92.7 \%$. The limit of quantitation (24) was confirmed at $2.5 \mathrm{ng} / \mathrm{mL}$. The coefficient of correlation $\left(r^{2}\right)$ was 0.9995 for the linear range of $2.5-500 \mathrm{ng} / \mathrm{mL}$. The intra-day and inter-day coefficients of variation were determined to be 5.6 and $10.7 \%$.

\section{Efficacy of Tiamulin in Neutropenic Chicken Intratracheal Infection Model}

To evaluate the efficacy of tiamulin at $24 \mathrm{~h}$ post-three day infections, either $0.85 \% \mathrm{NaCl}$ (controls) or tiamulin at 5, 10, 20, $30,40,50,60,70$, or $80 \mathrm{mg} / \mathrm{kg}$ were administrated to infected neutropenic chickens intramuscularly once daily for 3 days (five chickens/dose). At $24 \mathrm{~h}$ after the last drug administration, the amounts of $M$. gallisepticum in each chicken were calculated using the method described above.

\section{Pharmacokinetics and Pharmacodynamics Analysis}

The PK profiles of tiamulin were analyzed by the non-compartmental model with uniform weighting using the WinNonlin software (version 6.1; Pharsight, CA, USA). The surrogate marker of antibacterial activity, $\mathrm{AUC}_{24 \mathrm{~h}} / \mathrm{MIC}$ were calculated using in vitro MIC value and PK parameters obtained from three doses of i.m. administrations of tiamulin. The bacteria loading for each animal was calculated according to $C_{t}$ values and the in vitro standard DNA curve. The efficacy of tiamulin was evaluated by the reduction of $M$. gallisepticum compared to the initial bacteria count before drug treatment. The in vivo $\mathrm{PK} / \mathrm{PD}$ relationship of tiamulin against $M$. gallisepticum was studied using the sigmoid
$E_{\max }$ model WINNONLIN software (version 6.1; Pharsight, CA, USA) with the equation as follows:

$$
E=E_{0}+\frac{E_{\max } \times C_{e}^{N}}{\mathrm{EC}_{50}^{N}+C_{e}^{N}}
$$

where $E_{0}$ is the change in $\log _{10} \mathrm{ccu}$ equivalents $/ \mathrm{mL}$ in the control sample (absence of tiamulin), $E_{\max }$ is the difference in $\log _{10} \mathrm{ccu}$ equivalents $/ \mathrm{mL}$ of the greatest amount of kill, $C_{e}$ is the $\mathrm{AUC}_{24 \mathrm{~h}} /$ MIC in the effect compartment, $\mathrm{EC}_{50}$ is the $\mathrm{AUC}_{24 \mathrm{~h}} / \mathrm{MIC}$ value producing a $50 \%$ reduction in bacterial counts, and $N$ is the Hill coefficient that describes the steepness of the curve (25).

\section{Dosage Calculation}

In order to deduce a more rational regimen, the general formula was employed to estimate dosages for different magnitudes of efficiency (26).

$$
\text { Dose }=\frac{\mathrm{CL}_{\text {perhour }} \times\left(\mathrm{AUC}_{24} / \mathrm{MIC}\right) \times \mathrm{MIC}_{90}}{F \times f_{u}}
$$

where dose is the optimal dose (milligram/kilogram/day), CL is the body clearance (liter/kilogram/day), AUC/MIC is the breakpoint marker for the desired effect (hour), $\mathrm{MIC}_{90}$ is the MIC inhibiting $90 \%$ of strains (milligram/liter), $F$ is the bioavailability, and $f_{u}$ is the free drug fraction.

\section{RESULTS}

\section{Susceptibility Testing}

The MIC of tiamulin against the studied strain was $0.03 \mu \mathrm{g} / \mathrm{mL}$.

\section{In Vitro Standard DNA Curve}

The correlation between the $C_{\mathrm{t}}$ values and $\log _{10} \mathrm{ccu} / \mathrm{mL}$ reached statistic significance with the equation of $y=-0.3087 x+10.44$ and $R^{2}$ of 0.9988 (Figure 1). The limitation of detection was $3 \times 10^{2}$ equivalents $\mathrm{ccu} / \mathrm{mL}$. The recovery rates at different dilutions were $56.5 \pm 5.1 \%$.

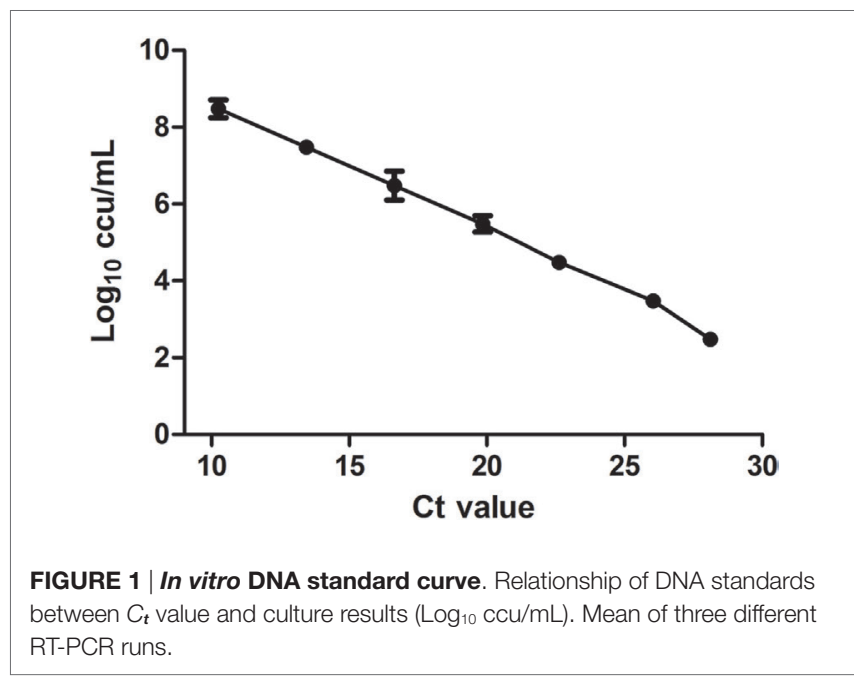




\section{M. gallisepticum Intratracheal Infection Model}

Major clinical symptoms, including depression, mouth breathing, eye closures, were observed from the infected animals. In addition, histopathology studies showed that airsacculitis as the cardinal symptom of $M$. gallisepticum infection was observed in $95 \%$ infected chickens. The mean M. gallisepticum load was $2.2 \times 10^{6} \mathrm{ccu}$ equivalents/mL for all inoculated chickens. The morbidity and mortality rates were 95 and $17 \%$ at 5 days postinfection, respectively. Neither clinical symptoms nor airsacculitis were observed in the control animals. Bacteriological assays were also negative in the control group.

\section{Tiamulin Pharmacokinetics Profiles in Neutropenic Intratracheal Infection Model}

The main PK parameters are presented in Table $\mathbf{1}$ and Figure 2. The $C_{\max }$ were $2.05,8.8$, and $14 \mu \mathrm{g} / \mathrm{mL}$ for 5,40 , and $80 \mathrm{mg} / \mathrm{kg}$ doses, respectively, which were observed at $0.167 \mathrm{~h}$ after administration. The half-life $\left(T_{1 / 2 \beta}\right)$ was about $1.24 \mathrm{~h}$ for all three different doses. A second peak was observed for all the doses administered at 8-12 h. Importantly, a significant correlation between doses and $\mathrm{AUC}_{24 \mathrm{~h}}$ was observed $\left(R^{2}=0.999\right.$, Figure 3$)$. As the $\mathrm{AUC}_{24 \mathrm{~h}}$ were increasing in a dose-dependent manner from 5 to $80 \mathrm{mg} / \mathrm{kg}$, the $\mathrm{AUC}_{24 \mathrm{~h}}$ of other dose regimens were calculated according to the linear relationship. The parameters of CL were parameterized as $\mathrm{CL} / \mathrm{F}$ because of the extravascular administration while $\mathrm{MRT}_{\text {last }}$ was the ratio of $\mathrm{AUMC}_{\text {last }} / \mathrm{AUC}_{\text {last }}$.

\section{In Vivo Efficacy of Tiamulin in Neutropenic Chicken Intratracheal Infection Model}

The $C_{t}$ values of respiratory tissues of chickens administrated with doses from 5 to $80 \mathrm{mg} / \mathrm{kg}$ increased with the increasing of doses, implying that the bacteria loading decreased with the dose increasing. The $C_{t}$ value increased sharply between dosages of 10 and $50 \mathrm{mg} / \mathrm{kg}$ while gently from 50 to $80 \mathrm{mg} / \mathrm{kg}$ (Figure 4). Limbs Twitch was observed in chickens treated with doses of 60,70 , $80 \mathrm{mg} / \mathrm{kg}$ for about $10 \mathrm{~min}$; however, no death emerged.

\section{Tiamulin PK/PD Profiles}

The $\mathrm{PK} / \mathrm{PD}$ indices $\mathrm{AUC}_{24 \mathrm{~h}} / \mathrm{MIC}$ was integrated using the $\mathrm{PK}$ parameters, dose proportionality, and MIC data. The effect $(E)$ was calculated as the reduction of M. gallisepticum using the

TABLE 1 | Pharmacokinetic parameters of tiamulin in serum following intramuscular administration at a single dose of 5,40 , or $80 \mathrm{mg} / \mathrm{kg}$ in M. gallisepticum-infected chickens ( $n=10 /$ group).

\begin{tabular}{|c|c|c|c|}
\hline Parameters (units) & $5 \mathrm{mg} / \mathrm{kg}$ & 40 mg/kg & $80 \mathrm{mg} / \mathrm{kg}$ \\
\hline$C_{\max }(\mu \mathrm{g} / \mathrm{mL})$ & 2.05 & 8.8 & 14 \\
\hline$T_{\max }(\mathrm{h})$ & 0.167 & 0.167 & 0.167 \\
\hline$T_{1 / 2 \beta}(\mathrm{h})$ & 1.15 & 1.39 & 1.17 \\
\hline 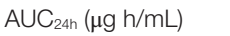 & 1.07 & 8.61 & 18.21 \\
\hline $\mathrm{CL} / \mathrm{F}(\mathrm{L} / \mathrm{h} / \mathrm{kg})$ & 4.53 & 4.6 & 4.37 \\
\hline $\mathrm{MRT}_{\text {last }}(\mathrm{h})$ & 8.15 & 3.46 & 2.33 \\
\hline
\end{tabular}

$C_{\max }$ maximum serum concentration; $T_{\max }$ time of maximum serum concentration; $T_{1 / 2 \beta}$ elimination half-life; $A \cup C_{24 h}, 24 \mathrm{~h}$ area under serum concentration-time curve; $C L / F$, clearance divided by bioavailability; MRT last, residence time. unit of $\log _{10} \mathrm{ccu}$ equivalent/mL. The relationship between the ratio of $\mathrm{AUC}_{24 \mathrm{~h}} / \mathrm{MIC}$ and antibiotic efficacy was described using the Sigmoid $E_{\max }$ model (Figure 5). The $\mathrm{AUC}_{24 \mathrm{~h}} / \mathrm{MIC}$ corrected well with the effects $\left(R^{2}=0.8979\right)$. The values of $\mathrm{AUC}_{24 \mathrm{~h}} / \mathrm{MIC}$ for mycoplasmastasis ( $0 \log _{10} \mathrm{ccu} / \mathrm{mL}$ reduction), for $1 \log _{10} \mathrm{ccu}$ reduction and $2 \log _{10}$ ccu reduction are 98.98, 206.56, 382.58, respectively (Table 2 ). The $\mathrm{EC}_{50}$ was $211.19 \mathrm{~h}$, and the slope of the graph $(N)$ was 3.68 .

\section{Dosage Calculation}

To calculate dosages, the bioavailability was taken into account owing to the extravascular route of administration, and the free drug fraction was not required for using PD data generated in vivo. In order to provide a dosage regimen attaining maximum effect for chickens infected with $M$. gallisepticum with an $\mathrm{MIC}_{90}$ of $0.03 \mu \mathrm{g} / \mathrm{mL}$, a dose of $45 \mathrm{mg} / \mathrm{kg}$ for 3 days was recommended.

\section{DISCUSSION}

M. gallisepticum, a highly transmissible, persistent pathogen in chickens, turkeys, and some wild birds, causes considerable economical losses to the poultry industry all over the world (27). Burch and Valks (28) had clarified that tiamulin possessed excellent activity against $241 \mathrm{M}$. gallisepticum strains by comparing the MIC of tiamulin against M. gallisepticum and the $C_{\max }$ or concentration at steady state $\left(C_{s s}\right)$ in chicken blood in 2002 (28). However, its chemotherapeutic properties are not well described. In addition, the use of PK/PD model to identify the PD activity by integrating the PK characters, MIC, and pathogen loading outcome has proven helpful in designing rational dosage regimens in humans and animals $(29,30)$. Burch and Alvarez (31) evaluated the relationship between $C_{\max } / \mathrm{MIC}$ or AUC/MIC and prevention or treatment results of tiamulin in M. gallisepticum infection in 3-week-old chickens (31). The PK/PD parameters in his study were with some rough as he used the reported PKs of tiamulin in 7-week-old health chickens to predict that in 3-week-old M. gallisepticum-infected chickens. And the PDs of that study was evaluated by scores of lesions, and whether M. gallisepticum was isolated or not from air sacs. It is known that isolation of M. gallisepticum is easy to influence by overgrowth of faster growing Mycoplasma species or impeded by other organisms or no growth in subculture, so it may not be a good method to judge the PDs. What's more, the study did not calculate the PDs from the aspect of bacteria loading reduction, which is important in PK/PD study. Thus, in this study, in vivo PK/PD profiles of tiamulin were established using PK characters in infected chickens, MIC, and pathogenloading outcome.

This was the first report about the PKs of tiamulin in M. gallisepticum intratracheal-infected chicks. Tiamulin was rapidly absorbed with the peak concentrations for $5,40,80 \mathrm{mg} /$ $\mathrm{kg}$ dose of $2.05,8.8,14.0 \mu \mathrm{g} / \mathrm{mL}$ achieved at $0.167 \mathrm{~h}$. The half-life was in the range of $1.15-1.39 \mathrm{~h}$, which was lower than the value reported in dogs $(4.7 \mathrm{~h})$ (32), which indicated that the elimination of tiamulin was of significant difference among species. The rate and extent of absorption was constant among the tested doses with the values of clearance divided by bioavailability 


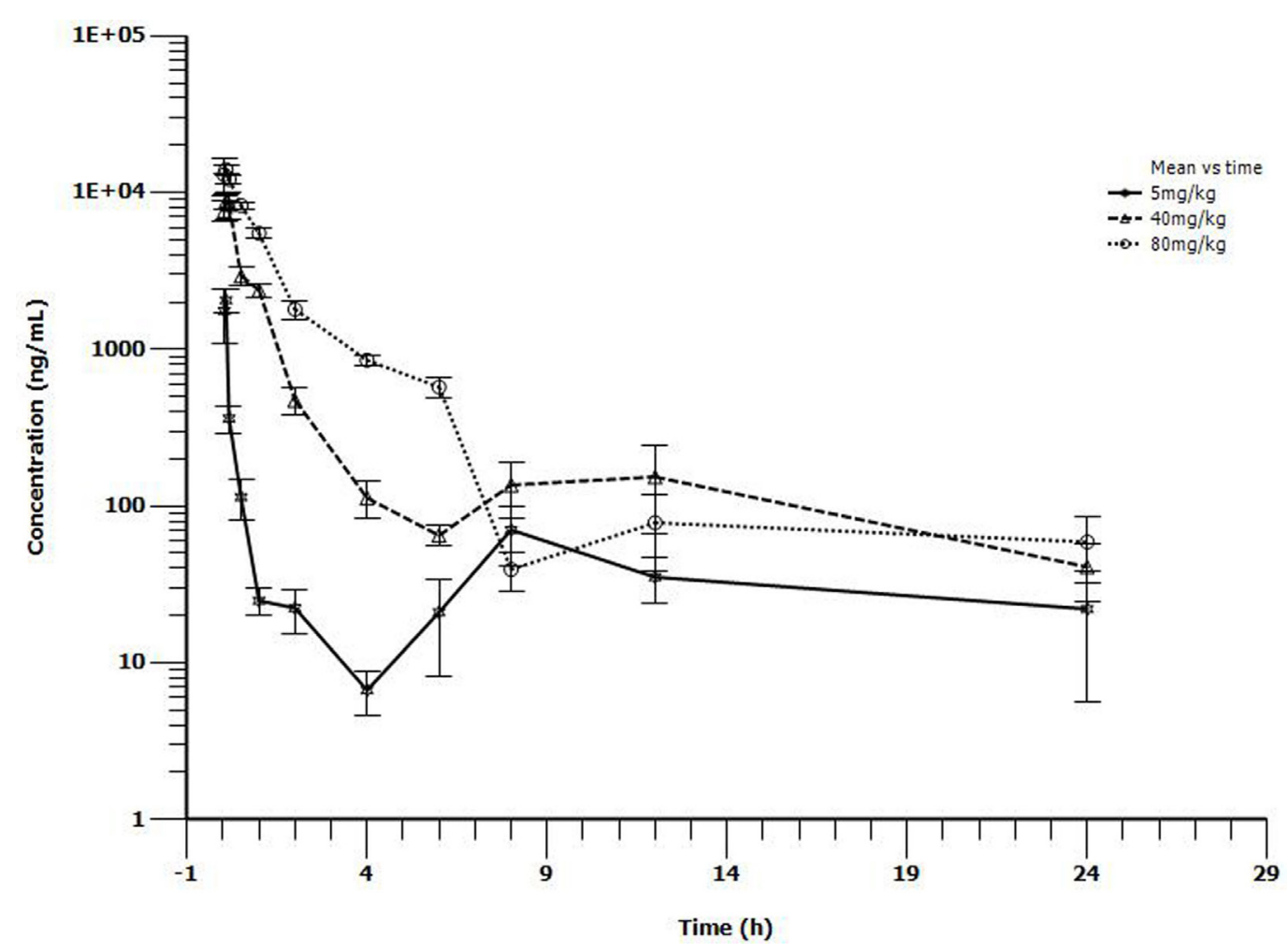

FIGURE 2 | Serum concentrations of tiamulin following i.m. administration at a single dose of 5, 40, or 80 mg/kg in $M$. gallisepticum intratracheal infection model ( $n=10 /$ time point).

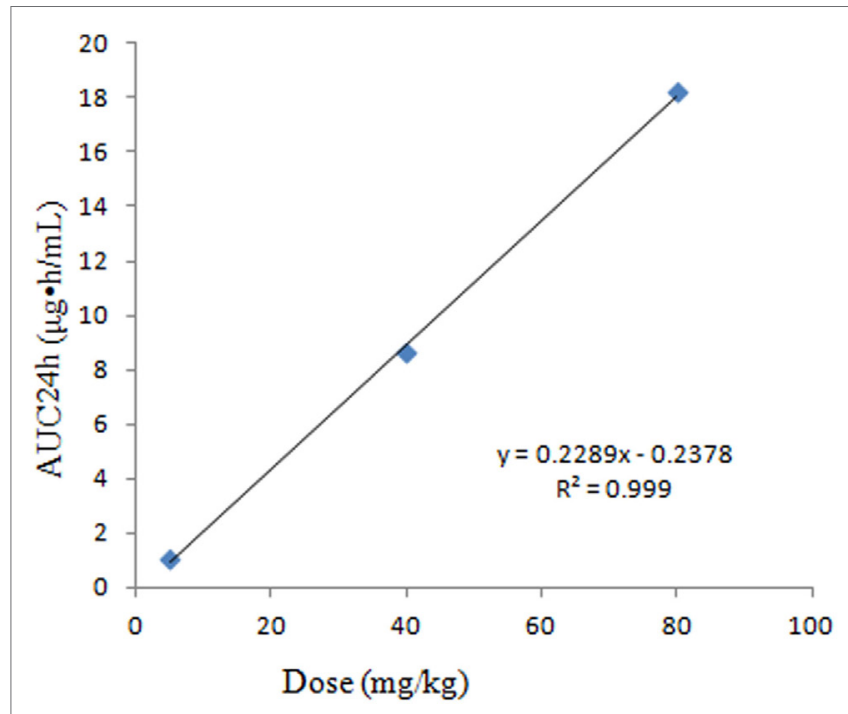

FIGURE 3 | Linear regression plots between administered dose and $\mathrm{AUC}_{24 \mathrm{~h}}$ values.

(CL/F) of $4.53,4.6,4.37 \mathrm{~L} / \mathrm{h} / \mathrm{kg}$, respectively. That is to say, tiamulin showed dose-dependent PKs when given as a single i.m. dose of 5,45 , or $80 \mathrm{mg} / \mathrm{kg}$ b.w. This was in accordance with the report that tiamulin showed dose proportionality in the range

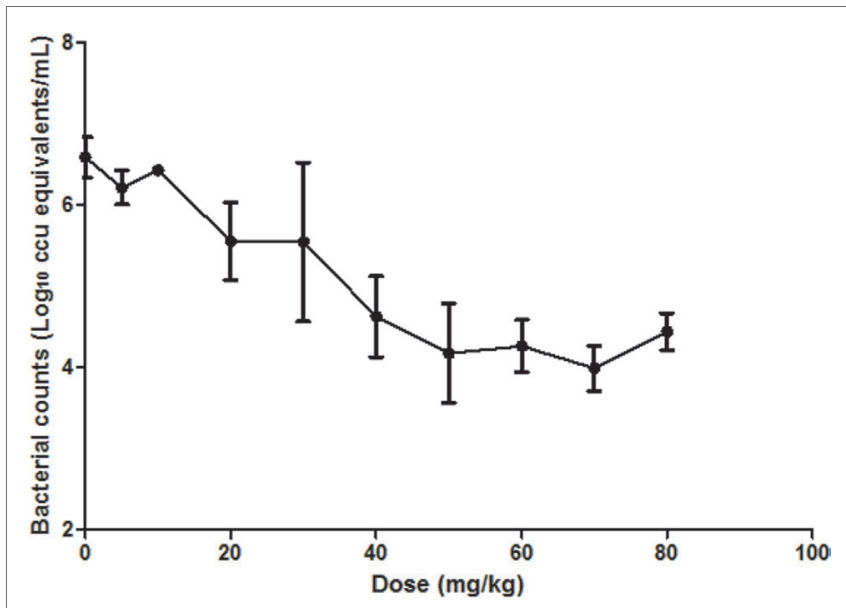

FIGURE 4 | The calculated in vivo M. gallisepticum counts after tiamulin treatment. ( $n=4-7 /$ dose).

of $10-25 \mathrm{mg} / \mathrm{kg}$ b.w. in dogs (32). This phenomenon was also confirmed in our previously research with valnemulin, which is also a semisynthetic derivative of the diterpene antibiotic pleuromutilin (19). A second peak was observed for all the doses administration at 8-12 h. Tiamulin mainly accumulates and metabolizes via liver. It was speculated that, according to the chemical structure, a relatively high liposolubility of tiamulin 


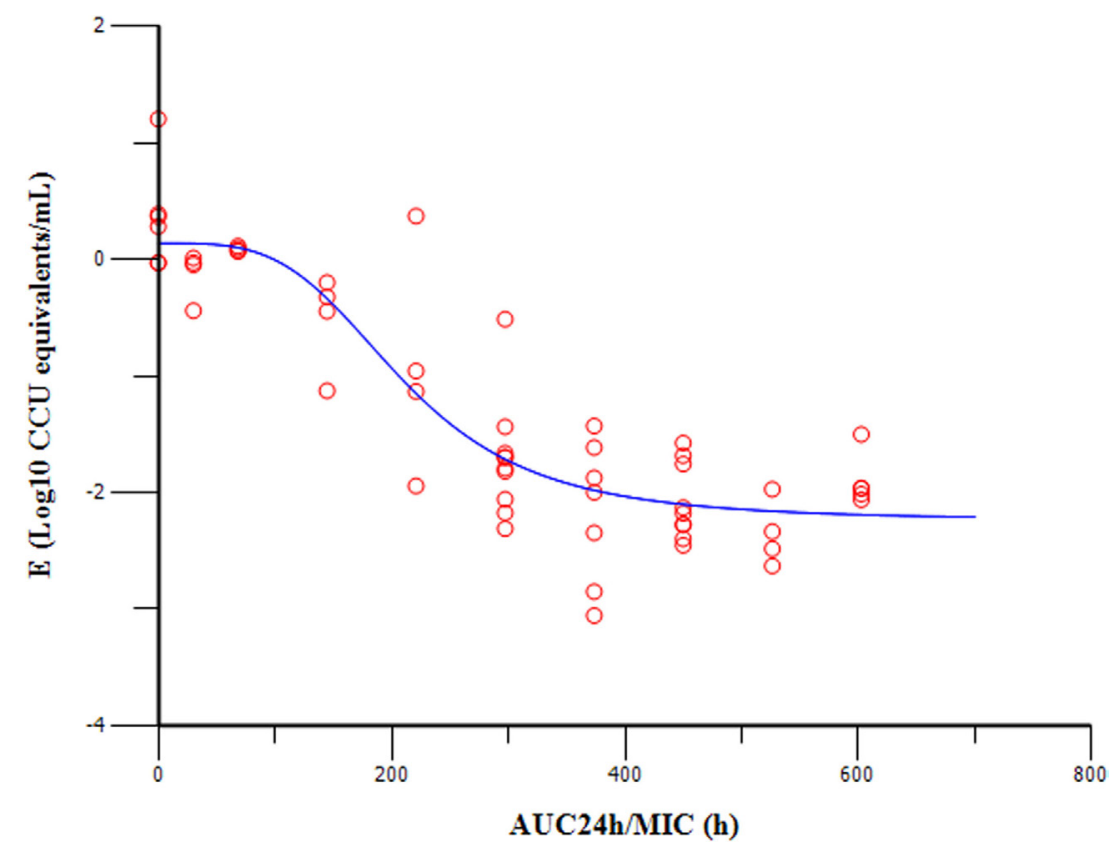

FIGURE 5 | Sigmoid $E_{\max }$ relationships of tiamulin between antimycoplasmal effect $\left(E, \log _{10} \mathrm{ccu}\right.$ equivalents/mL) and in vivo AUC ${ }_{24 h} / \mathrm{MIC}$ ratio against MG in serum of chickens.

TABLE 2 | Tiamulin PK/PD analysis with the parameter of $\mathrm{AUC}_{24 \mathrm{~h}} / \mathrm{MIC}$ in M. gallisepticum intratracheal infection model.

\begin{tabular}{lrr}
\hline Parameter & Value & SD \\
\hline$E_{\max }\left(\log _{10}\right.$ Ccu equivalents $\left./ \mathrm{mL}\right)$ & 2.38 & 0.24 \\
$E_{0}\left(\mathrm{Log}_{10} \mathrm{Ccu}\right.$ equivalents $\left./ \mathrm{mL}\right)$ & 0.14 & 0.13 \\
$\mathrm{EC}_{50}(\mathrm{~h})$ & 211.19 & 20.3 \\
$\mathrm{AUC}$ & 98.98 & \\
$\mathrm{AUC}_{24 \mathrm{~h}} / \mathrm{MIC}$ for $0 \log _{10} \mathrm{Cc}$ IC for $1 \log _{10} \mathrm{Ccu}$ equivalents $/ \mathrm{mL}$ reduction $(\mathrm{h})$ & 206.56 & \\
$\mathrm{AUC}_{24 \mathrm{~h}} / \mathrm{MIC}$ for $2 \log _{10} \mathrm{Ccu}$ equivalents $/ \mathrm{mL}$ reduction $(\mathrm{h})$ & 382.58 & \\
Slope $(\mathrm{N})$ & 3.68 & 1.2 \\
\hline
\end{tabular}

$E_{0}$ is the change in $\log _{10}$ ccu equivalents/ $\mathrm{mL}$ after $24 \mathrm{~h}$ incubation in the control sample (absence of drug) compared with the initial inoculum. $E_{\max }$ is the difference in effect of the greatest amount of kill. $E C_{50}$ is the $A U C_{24 h} / M I C$ value producing a $50 \%$ reduction in bacterial counts from the initial inoculum. $A \cup C_{24 h} / M I C$ is the $24 \mathrm{~h}$ area under concentration-time curve/minimum inhibitory concentration ratios. $N$ is the Hill coefficient that describes the steepness of the $A \cup C_{24 i} / M I C$-effect curve.

would result in enterohepatic recycling, which is possibly the main reason that the second peak was observed (33).

The parameters of tiamulin $\left(\mathrm{AUC}_{24 \mathrm{~h}}\right)$ that showed dose proportionality in the range of $5-80 \mathrm{mg} / \mathrm{kg}$ following i.m. administration allowed us to calculate the $\mathrm{AUC}_{24 \mathrm{~h}}$ for other dosage administrations. As reported for other pleuromutilin derivatives $(34,35)$, the $\mathrm{PK} / \mathrm{PD}$ surrogate of pleuromutilin derivatives is $\mathrm{AUC}_{24 \mathrm{~h}} / \mathrm{MIC}$. The data from the present multiple dosage studies of tiamulin confirmed the conclusion that the $\mathrm{AUC}_{24 \mathrm{~h}} / \mathrm{MIC}$ was the PK/PD index of tiamulin. The values of $\mathrm{AUC}_{24 \mathrm{~h}} / \mathrm{MIC}$ for mycoplasmastasis $\left(0 \log _{10} \mathrm{ccu}\right.$ equivalents reduction), activity of $1 \log _{10} \mathrm{ccu}$ equivalents reduction, and $2 \log _{10} \mathrm{ccu}$ equivalents reduction were 124, 205, and $327 \mathrm{~h}$, respectively, which were much lower than those obtained from an in vivo study of valnemulin $(28,820,38,030$, and $56,256 \mathrm{~h}$, respectively) (36). One possible reason for the differences was that the concentration in serum was not that real one act on M. gallisepticum as the infection site is air sac or respiratory system, and antibiotic concentrations in air sac or lung are usually different from those in serum (14). Another reason could be that the distribution volume of timulin was much wider than that of valnemulin in chickens, which means that the tissue concentration of tiamulin maybe higher than that of valnemulin (36). Tiamulin did not reach a mycoplasmacidal effect ( $3 \log _{10} \mathrm{ccu}$ equivalents reduction), which was in accordance with Burch and Alvarez's (31) results that even tiamulin concentration far exceeded the MBC could not eliminate the pathogen in treatment procedure (31).

As was observed in this investigation, doses over $60 \mathrm{mg} / \mathrm{kg}$ would trigger neuro-toxicity. The calculated dose of $45 \mathrm{mg} / \mathrm{kg}$ for 3 days was sufficient for clinical treatment of $M$. gallisepticum infection with an $\mathrm{MIC}_{90}$ of $0.03 \mu \mathrm{g} / \mathrm{mL}$. This regimen was in the range of recommended doses for drinking water $(30-60 \mathrm{mg} / \mathrm{kg}$ ) in poultry but more than twice of the recommended dose range for i.m. administration (10-20 mg/kg) in pigs (13). It was also lower than the neuro-toxicity dose. Though the i.m. administration has not been approved for tiamulin in chicks, this result would provide foundation for tiamulin injectable formulation for chicks in future.

The conventional method for evaluating the efficacy of an antibacterial agent is bacteria counting by culture method. However, the cultivation technique is expensive and laborious and time consuming (37). Also, this gold standard for M. gallisepticum was usually influenced by overgrowth of faster 
growing Mycoplasma species or impeded by other organisms or no growth in subculture. The RT-PCR had been confirmed in a previous study for qualitative and quantitative detection of $M$. gallisepticum (36). The specificity, sensitivity, and reproducibility were sufficient for quantitative detection of $M$. gallisepticum in clinical samples (20).

Neutropenic chickens were used in this study to evade host immunity factors that may play important roles in the M. gallisepticum infections as well as the efficacy of antimicrobial therapy. A previous study suggested that the $\mathrm{PK} / \mathrm{PD}$ index magnitude necessary for successful therapy is reduced in animal models in the presence of neutrophils (38). Therefore, further studies on elucidation of host immunity-pathogen interactions are essential for the drug. Furthermore, a population PK approach should be conducted in the future to derive a population clearance of tiamulin for use in dose calculation. In addition, MIC distribution should be evaluated to take the variation of susceptibility of M. gallisepticum to tiamulin into account.

The present study characterized the in vivo activity of tiamulin against M. gallisepticum in a neutropenic chicken model. The $\mathrm{PK} / \mathrm{PD}$ surrogate $\mathrm{AUC}_{24 \mathrm{~h}} / \mathrm{MIC}$ correlated well with the in vivo antibacterial efficacy. The in vivo data suggest that animal dosage

\section{REFERENCES}

1. Adelman JS, Kirkpatrick L, Grodio JL, Hawley DM. House finch populations differ in early inflammatory signaling and pathogen tolerance at the peak of Mycoplasma gallisepticum infection. Am Nat (2013) 181(5):674-89. doi:10.1086/670024

2. Abolnik C, Gouws J. Extended survival times of Mycoplasma gallisepticum and Mycoplasma synoviae on kanekalon synthetic hair fibres. Poult Sci (2014) 93(1):8-11. doi:10.3382/ps.2013-03457

3. Dhondt AA, DeCoste JC, Ley DH, Hochachka WM. Diverse wild bird host range of Mycoplasma gallisepticum in eastern North America. PLoS One (2014) 9(7):e103553. doi:10.1371/journal.pone.0103553

4. Mohammed HO, Carpenter TE, Yamamoto R. Economic impact of Mycoplasma gallisepticum and M. synoviae in commercial layer flocks. Avian Dis (1987) 31(3):477-82. doi:10.2307/1590726

5. Kleven SH. Control of avian mycoplasma infections in commercial poultry. Avian Dis (2008) 52(3):367-74. doi:10.1637/8323-041808-Review.1

6. Peebles ED, Jacob R, Branton SL, Evans JD, Leigh SA, Gerard PD. Effects of different vaccine combinations against Mycoplasma gallisepticum on the digestive and reproductive organ characteristics of commercial egg-laying hens. Poult Sci (2015) 94(12):2898-904. doi:10.3382/ps/pev269

7. Lin H, Tian Y, Zhang Z, Wu L, Chen Y. Quantification of piperazine phosphate in human plasma by high-performance liquid chromatography-electrospray ionization tandem mass spectrometry employing precolumn derivatization with dansyl chloride. Anal Chim Acta (2010) 664(1):40-8. doi:10.1016/j. aca.2010.02.003

8. Stipkovits L, Burch DGS. Antibiotic resistance of mycoplasmas of chickens and turkey origin. Page 179 in Proceedings Xth World Veterinary Poultry Association Congress, Sydney, Australia. Sydney, Australia: Australian Veterinary Poultry Association (1993). Abstr. 121.

9. Bradbury JM, Yavari CA, Giles CJ. In vitro evaluation of various antimicrobials against Mycoplasma gallisepticum and Mycoplasma synoviae by the micro-broth method, and comparison with a commercially-prepared test system. Avian Pathol (1994) 23(1):105-15. doi:10.1080/03079459408418978

10. Hannan PC, Windsor GD, de Jong A, Schmeer N, Stegemann M. Comparative susceptibilities of various animal-pathogenic mycoplasmas to fluoroquinolones. Antimicrob Agents Chemother (1997) 41(9):2037-40.

11. Lin MY. In vitro comparison of the activity of various antibiotics and drugs against new Taiwan isolates and standard strains of avian mycoplasma. Avian Dis (1987) 31(4):705-12. doi:10.2307/1591020 regimens should supply $\mathrm{AUC}_{24 \mathrm{~h}} / \mathrm{MIC}$ of tiamulin of $382.68 \mathrm{~h}$ for $2 \log _{10}$ ccu equivalents $M$. gallisepticum reduction. These studies suggest that tiamulin, if used for treatment of $M$. gallisepticum infection with an $\mathrm{MIC}_{90}$ of $0.03 \mu \mathrm{g} / \mathrm{mL}$, would benefit from $45 \mathrm{mg} / \mathrm{kg}$ i.m. once daily for 3 days.

\section{AUTHOR CONTRIBUTIONS}

$\mathrm{XX}$, JS, and Y-HL designed this study; TY, XF, JC, and XX carried out the whole experiments; YX helped to analysis the data and revised this manuscript; XX and JS analyzed the data and write this manuscript and Y-HL revised it.

\section{FUNDING}

This study was supported by a research program for Changjiang Scholars, Innovative Research Team in University of Ministry of Education of China (Grant IRT13063), Science and Technology Planning Project of Guangdong Province China (Grant 2012A 020800004). The funders had no role in study design, data collection, and analysis, decision to publish, or preparation of the manuscript.

12. Gerchman I, Lysnyansky I, Perk S, Levisohn S. In vitro susceptibilities to fluoroquinolones in current and archived Mycoplasma gallisepticum and Mycoplasma synoviae isolates from meat-type turkeys. Vet Microbiol (2008) 131(3-4):266-76. doi:10.1016/j.vetmic.2008.04.006

13. EMA. (1999). Available at: http://www.ema.europa.eu/docs/en_GB/document library/Maximum_Residue_Limits_-_Report/2009/11/WC500015563.pdf

14. Pakpinyo S, Sasipreeyajan J. Molecular characterization and determination of antimicrobial resistance of Mycoplasma gallisepticum isolated from chickens. Vet Microbiol (2007) 125(1-2):59-65. doi:10.1016/j.vetmic.2007.05.011

15. Pridmore A. Antibacterial Activity of Tiamulin, Valnemulin, Tylosin and Lincomycin against Brachyspira and Mycoplasma Isolates: Determination of Minimum Inhibitory Concentration (MIC). Report to Novartis (2008).

16. Andes D, Craig WA. Animal model pharmacokinetics and pharmacodynamics: a critical review. Int J Antimicrob Agents (2002) 19(4):261-8. doi:10.1016/ S0924-8579(02)00022-5

17. Rogers GB, Hoffman LR, Doring G. Novel concepts in evaluating antimicrobial therapy for bacterial lung infections in patients with cystic fibrosis. J Cyst Fibros (2011) 10(6):387-400. doi:10.1016/j.jcf.2011.06.014

18. Hannan PC. Guidelines and recommendations for antimicrobial minimum inhibitory concentration (MIC) testing against veterinary Mycoplasma species. International Research Programme on Comparative Mycoplasmology. Vet Res (2000) 31(4):373-95. doi:10.1051/vetres:2000100

19. Xiao X, Sun J, Yang T, Fang X, Wu D, Xiong YQ, et al. In vivo pharmacokinetic/ pharmacodynamic profiles of valnemulin in an experimental intratracheal Mycoplasma gallisepticum infection model. Antimicrob Agents Chemother (2015) 59(7):3754-60. doi:10.1128/AAC.00200-15

20. Mekkes DR, Feberwee A. Real-time polymerase chain reaction for the qualitative and quantitative detection of Mycoplasma gallisepticum. Avian Pathol (2005) 34(4):348-54. doi:10.1080/03079450500179954

21. Craig WA, Andes DR. In vivo pharmacodynamics of ceftobiprole against multiple bacterial pathogens in murine thigh and lung infection models. Antimicrob Agents Chemother (2008) 52(10):3492-6. doi:10.1128/AAC. 01273-07

22. Hu F, Bian K, Liu Y, Su Y, Zhou T, Song X, et al. Development of a modified QUick, Easy, CHeap, Effective, Rugged and Safe method for the determination of multi-class antimicrobials in vegetables by liquid chromatography tandem mass spectrometry. J Chromatogr A (2014) 1368:52-63. doi:10.1016/j. chroma.2014.09.074

23. Hu FY, He LM, Yang JW, Bian K, Wang ZN, Yang HC, et al. Determination of 26 veterinary antibiotics residues in water matrices by lyophilization in 
combination with LC-MS/MS. J Chromatogr B Analyt Technol Biomed Life Sci (2014) 94(9-950):79-86. doi:10.1016/j.jchromb.2014.01.008

24. Patel N, Pai MP, Rodvold KA, Lomaestro B, Drusano GL, Lodise TP. Vancomycin: we can't get there from here. Clin Infect Dis (2011) 52(8):969-74. doi:10.1093/cid/cir078

25. Burton ME, Shaw LM, Schentag JJ, Evans WE. Applied Pharmacokinetics \& Pharmacodynamics: Principles of Therapeutic Drug Monitoring. Philadelphia: Lippincott Williams and Wilkins Press (2005). p. 61-5.

26. Toutain PL, Bousquet-Melou A, Martinez M. AUC/MIC: a PK/PD index for antibiotics with a time dimension or simply a dimensionless scoring factor? J Antimicrob Chemother (2007) 60(6):1185-8. doi:10.1093/jac/dkm360

27. Evans JD, Leigh SA, Branton SL, Collier SD, Pharr GT, Bearson SMD. Mycoplasma gallisepticum: current and developing means to control the avian pathogen. J Appl Poult Res (2005) 14:757-63. doi:10.1093/japr/14.4.757

28. Burch DGS, Valks M. Comparison of minimal inhibitory concentrations (Mic) against chicken mycoplasma of tiamulin and other antimicrobials and their concentrations in the blood. Proceedings of the 12th World Veterinary Poultry Congress. Cairo, Egypt: (2002). 322 p.

29. Andes D, Craig WA. In vivo activities of amoxicillin and amoxicillinclavulanate against Streptococcus pneumoniae: application to breakpoint determinations. Antimicrob Agents Chemother (1998) 42(9):2375-9.

30. Craig WA. Pharmacokinetic/pharmacodynamic parameters: rationale for antibacterial dosing of mice and men. Clin Infect Dis (1998) 26(1): 1-10; quiz 11-12. doi:10.1086/516284

31. Burch DGS, Alvarez R. Cmax \& AUC/MIC (AUIC) relationships for tiamulin prevention and treatment of Mycoplasma gallisepticum infections in chickens. The 2nd International Conference on Antimicrobial Agents in Veterinary Medicine. Ottawa, Canada: (2004). 29 p.

32. Laber G. Investigation of pharmacokinetic parameters of tiamulin after intramuscular and subcutaneous administration in normal dogs. J Vet Pharmacol Ther (1988) 11(1):45-9. doi:10.1111/j.1365-2885.1988.tb00119.x
33. Mostafavi SA, Foster AT. A double-peak phenomenon in the pharmacokinetics of acebutolol enantiomers after oral administration: discontinuous absorption of acebutolol. Daru J Faculty Pharm (2002) 10(4):141-7.

34. Novak R. Are pleuromutilin antibiotics finally fit for human use? Ann N Y Acad Sci (2011) 1241:71-81. doi:10.1111/j.1749-6632.2011.06219.x

35. Zhao DH, Zhou YF, Yu Y, Shi W, Yang X, Xiao X, et al. Integration of pharmacokinetic and pharmacodynamic indices of valnemulin in broiler chickens after a single intravenous and intramuscular administration. Vet $J$ (2014) 201(1):109-15. doi:10.1016/j.tvjl.2014.05.010

36. Xiao X, Zhao DH, Yang X, Shi W, Deng H, Ma J, et al. Mycoplasma gallisepticum and Escherichia coli mixed infection model in broiler chickens for studying valnemulin pharmacokinetics. J Vet Pharmacol Ther (2014) 37(1):99-102. doi:10.1111/jvp.12065

37. Garcia M, Jackwood MW, Levisohn S, Kleven SH. Detection of Mycoplasma gallisepticum, M. synoviae, and M. iowae by multi-species polymerase chain reaction and restriction fragment length polymorphism. Avian Dis (1995) 39(3):606-16. doi:10.2307/1591815

38. Pechere M, Letarte R, Pechere JC. Efficacy of different dosing schedules of tobramycin for treating a murine Klebsiella pneumoniae bronchopneumonia. J Antimicrob Chemother (1987) 19(4):487-91. doi:10.1093/jac/19.4.487

Conflict of Interest Statement: The authors declare that the research was conducted in the absence of any commercial or financial relationships that could be construed as a potential conflict of interest.

Copyright $\odot 2016$ Xiao, Sun, Yang, Fang, Cheng, Xiong and Liu. This is an open-access article distributed under the terms of the Creative Commons Attribution License (CC BY). The use, distribution or reproduction in other forums is permitted, provided the original author(s) or licensor are credited and that the original publication in this journal is cited, in accordance with accepted academic practice. No use, distribution or reproduction is permitted which does not comply with these terms. 